\title{
Accuracy of enzyme-linked immunospot assay for diagnosis of pleural tuberculosis: a meta-analysis
}

\author{
Z.Z. Li ${ }^{1}$, W.Z. Qin ${ }^{1}$, L. Li ${ }^{1}$, Q. Wu ${ }^{1}$ and Y.J. Wang ${ }^{1,2}$ \\ ${ }^{1}$ West China School of Medicine/West China Hospital, Sichuan University, \\ Chengdu, Sichuan, China \\ ${ }^{2}$ Health Management Center, West China Hospital, Sichuan University, \\ Chengdu, Sichuan, China \\ Corresponding author: Y.J. Wang \\ E-mail: yjwang1963@sina.com
}

Genet. Mol. Res. 14 (3): 11672-11680 (2015)

Received March 19, 2015

Accepted May 25, 2015

Published September 28, 2015

DOI http://dx.doi.org/10.4238/2015.September.28.19

ABSTRACT. Current methods for diagnosing tuberculous pleurisy are poor. Some studies have explored the diagnostic value of a pleural effusion enzyme-linked immunospot (ELISPOT) assay, but its accuracy remains controversial. Therefore, we performed a meta-analysis of the existing evidence on the ability of the ELISPOT assay to diagnose tuberculous pleurisy. We systematically searched PubMed, Google Scholar, and EMBASE databases for studies measuring the sensitivity, specificity, and other measures of accuracy of the pleural effusion ELISPOT assay for diagnosis of tuberculous pleurisy. A total of nine studies were identified and subjected to meta-analysis, giving the following pooled values for diagnostic accuracy: sensitivity, 0.93 [95\% confidence intervals $(95 \% \mathrm{Cl})$ $=0.90$ to 0.95$]$; specificity, $0.90(95 \% \mathrm{Cl}=0.86$ to 0.93$)$; positive likelihood ratio, $8.21(95 \% \mathrm{Cl}=4.00$ to 16.84$)$; negative likelihood ratio, $0.11(95 \% \mathrm{Cl}$ $=0.06$ to 0.19$)$; diagnostic odds ratio, $88.26(95 \% \mathrm{Cl}=33.81$ to 230.43$)$; and the area under the curve, 0.9599 (standard error of the mean, 0.0134). The available evidence suggests that pleural effusion ELISPOT assay is 
sufficiently accurate to diagnose tuberculous pleurisy as a stand-alone technique. In fact, it appears to be superior to assays based on adenosine deaminase and gamma interferon for screening patients and confirming the diagnosis of tuberculous pleurisy.

Key words: Diagnosis; Enzyme-linked immunospot; Meta-analysis; Tuberculous pleurisy

\section{INTRODUCTION}

Tuberculosis causes high morbidity and mortality around the world (Müller et al., 2014). Although its global incidence appears to be declining (World Health Organization, 2009), its diagnosis remains challenging. Approximately $30 \%$ of tuberculosis cases present as tuberculous pleurisy (Aktogu et al., 1996), for which the available diagnostic methods have significant limitations. Culture of pleural fluid shows sensitivities of only 24 to $58 \%$ (Trajman et al., 2008; Porcel, 2009), while pleural tissue biopsy is invasive and technically difficult (Pérez-Rodriguez and Jiménez Castro, 2000).

As a less invasive and more reliable alternative, investigators have developed a pleural effusion enzyme-linked immunospot (ELISPOT) assay to diagnose tuberculous pleurisy. Despite extensive research on this assay, its diagnostic accuracy remains controversial. For example, one study reported a sensitivity of $95.7 \%$ and a specificity of $100 \%$ (Liao et al., 2014), while another study reported corresponding values of only 86 and $60 \%$ (Dheda et al., 2009). Since these accuracy studies were carried out with diverse sample sizes, patients of different ethnicities, and differing technical parameters (such as detection cut-offs), we wanted to assess the available evidence as rigorously and comprehensively as possible.

Therefore, we performed a meta-analysis of the available evidence to determine the accuracy of ELISPOT assay for diagnosis of tuberculous pleurisy.

\section{MATERIAL AND METHODS}

\section{Search strategy and study selection criteria}

After failing to find any meta-analyses on the accuracy of ELISPOT for diagnosis of tuberculous pleurisy, we searched for relevant individual studies published up to November 31, 2014, in PubMed, Google Scholar, and EMBASE databases, using the following search terms: "tuberculosis pleurisy" or "tuberculosis pleural effusion" and "enzyme-linked immunospot" or "t-spot". Reference lists of relevant articles were also searched manually.

To be included in our meta-analysis, studies had to satisfy the following criteria: 1) they had to be complete research articles, so abstracts, case reports and letters to the editor were excluded; 2) they had to use a case-control design and clearly report their diagnostic criteria; 3) they had to report at least sensitivity and specificity, together with $95 \%$ confidence intervals $(95 \% \mathrm{Cls})$ or the raw data needed to determine $95 \% \mathrm{Cls}$, such as the number of patients. Two reviewers (Z.L. and W.Q.) independently reviewed and assessed study eligibility, and disagreements were resolved by a third author (Y.W.).

\section{Data extraction and quality assessment}

Two investigators (Z.L. and W.Q.) independently extracted the following information 
from each study: first author name, country of origin, year of publication, numbers of patients and controls, assay methods, the type of sample detected, sensitivity and specificity data, and numbers of truly positive, false-positive, truly negatives, and false-negative. The methodological quality of each study was assessed using the criteria of the Quality Assessment of Diagnostic Accuracy Studies (QUADAS) (Whiting et al., 2003). This evidence-based quality assessment tool has a maximum score of 14 .

\section{Statistical analyses}

Analyses were performed using Stata, version 12 (Stata Corporation, College Station, TX, USA), and Meta-Disc (Zamora J, Muriel A, and Abraira V. Meta-DiSc for Windows, presented at the XI Cochrane Colloquium, Barcelona, Spain, 2003). Data from individual studies were pooled using a random-effect model and used to generate values for the following measures of test accuracy: sensitivity, specificity, positive likelihood ratio (PLR), negative likelihood ratio (NLR), diagnostic odds ratio (DOR), a summary receiver operating characteristic (SROC) curve (Chen et al., 2014), and the area underneath the SROC curve. Chi-square tests were used to assess heterogeneity among the included studies for the measures of test accuracy. Deeks funnel plots were used to assess the risk of publication bias (Deeks et al., 2005).

\section{RESULTS}

Systematic searching of the literature identified 22 potentially eligible studies, among which 10 were excluded based on a review of titles and abstracts. The full text of the remaining 10 articles was examined, and one study (Losi et al., 2010) was excluded because it did not use the t-spot assay format. The final meta-analysis therefore included nine publications (Losi et al., 2007; Lee et al., 2009; Dheda et al., 2009; Kang et al., 2012; Keng et al., 2013; Liao et al., 2014; Zhang et al., 2014; Liu et al., 2013, 2014). The clinical characteristics of each study, including QUADAS scores, are displayed in Table 1.

\begin{tabular}{|c|c|c|c|c|c|c|c|c|}
\hline Study first-author & Country (year) & No. of patients & Method $^{\mathrm{a}}$ & TP & FP & $\mathrm{FN}$ & $\mathrm{TN}$ & Quality score (QUADAS) \\
\hline Dheda & South Africa (2009) & 36 & ELISPOT & 31 & 6 & 5 & 9 & 12 \\
\hline Losi & Germany (2007) & 20 & ELISPOT & 19 & 5 & 1 & 16 & 13 \\
\hline Keng & Taiwan (2013) & 14 & ELISPOT & 10 & 1 & 4 & 37 & 11 \\
\hline Liu & China (2013) & 54 & ELISPOT & 52 & 2 & 2 & 40 & 12 \\
\hline Zhang & China (2013) & 45 & ELISPOT & 40 & 7 & 5 & 42 & 13 \\
\hline Liu & China (2014) & 59 & ELISPOT & 54 & 5 & 5 & 52 & 10 \\
\hline Liao & China (2014) & 281 & ELISPOT & 269 & 0 & 12 & 51 & 10 \\
\hline Lee & Taiwan (2008) & 19 & ELISPOT & 18 & 3 & 1 & 18 & 12 \\
\hline Kang & Korea (2012) & 21 & ELISPOT & 17 & 1 & 4 & 14 & 11 \\
\hline
\end{tabular}

$\mathrm{TN}=$ truly negative result; $\mathrm{TP}=$ truly positive result; $\mathrm{FN}=$ false-negative result; FP = false-positive result. ${ }^{\text {aEnzyme- }}$ linked immunospot assay (T-SPOT-TB; Oxford Immunotec Ltd., Oxford, UK) measuring the immune response to an ESAT-6 or CFP-10 peptide.

\section{Study characteristics and quality}

The total sample size in the nine studies was 858 , comprising 549 patients with tuberculous pleurisy and 309 without any tuberculosis. In all studies, ELISPOT assay was performed 
on mononuclear cells from pleural effusion, which had been stimulated with Mycobacterium tuberculosis-specific antigens early secretory antigenic target 6 and culture filtrate protein 10. All studies had a high QUADAS score.

\section{Diagnostic accuracy}

Data from all nine studies were subjected to a meta-analysis to generate pooled values for diagnostic accuracy parameters, as follows: sensitivity, $0.93(95 \% \mathrm{Cl}=0.90$ to 0.95$)$ (Figure 1); specificity, $0.90(95 \% \mathrm{Cl}=0.86$ to 0.93$)$ (Figure 2); PLR, $8.21(95 \% \mathrm{Cl}=4.00$ to 16.84$)$ (Figure 3); NLR, $0.11(95 \% \mathrm{Cl}=0.06$ to 0.19$)$ (Figure 4); DOR, $88.26(95 \% \mathrm{Cl}=33.81$ to 230.43$)$ (Figure 5); and area under the SROC curve, 0.9599 (standard error of the mean, 0.0134) (Figure 6). Data from the studies showed high heterogeneity for these accuracy parameters, based on $\mathrm{I}^{2}$ values of $54.4 \%$ for sensitivity, $73.1 \%$ for specificity, $75.6 \%$ for PLR, $65.7 \%$ for NLR, and $58.1 \%$ for DOR.
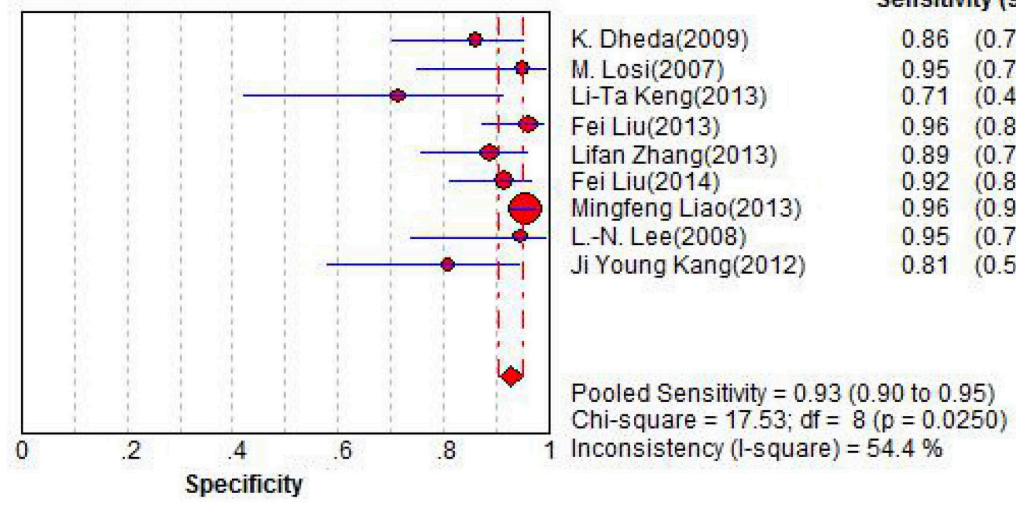

Figure 1. Forest plot of sensitivity estimates for the pleural fluid ELISPOT assay for the diagnosis of tuberculous pleurisy. Point estimates of sensitivity from each study are shown as solid circles, whose size reflects the total number of cases and controls. Error bars show $95 \% \mathrm{Cls}$. Numbers indicate the reference numbers of the studies.

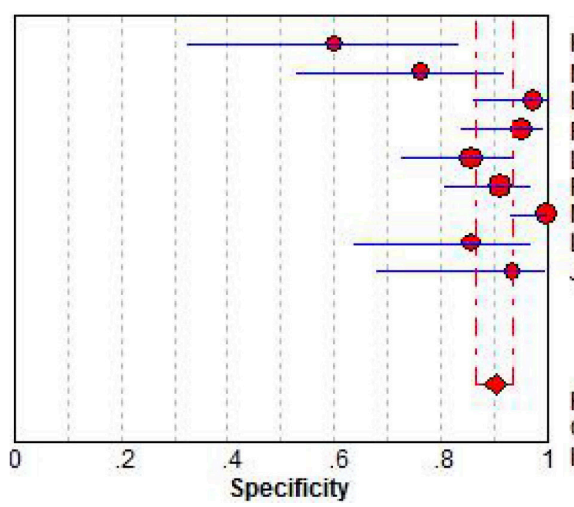

K. Dheda(2009)

M. Losi (2007)

Li-Ta Keng(2013)

Fei Liu(2013)

Lifan Zhang(2013)

Fei Liu(2014)

Mingfeng Liao(2013)

L.-N. Lee(2008)

Specificity $(95 \% \mathrm{Cl})$

$0.60 \quad(0.32-0.84)$

$0.76(0.53-0.92)$

$0.97 \quad(0.86-1.00)$

$0.95(0.84-0.99)$

$0.86(0.73-0.94)$

$0.91(0.81-0.97)$

$1.00(0.93-1.00)$

$0.86(0.64-0.97)$

$0.93(0.68-1.00)$

Pooled Specificity $=0.90(0.86$ to 0.93$)$

Chi-square $=29.70 ; \mathrm{df}=8(\mathrm{p}=0.0002)$

Inconsistency (I-square) $=73.1 \%$

Figure 2. Forest plot of specificity estimates for the pleural fluid ELISPOT assay for the diagnosis of tuberculous pleurisy. Point estimates of specificity from each study are shown as solid circles, whose size reflects the total number of cases and controls. Error bars show $95 \% \mathrm{Cls}$. Numbers indicate the reference numbers of the studies. 


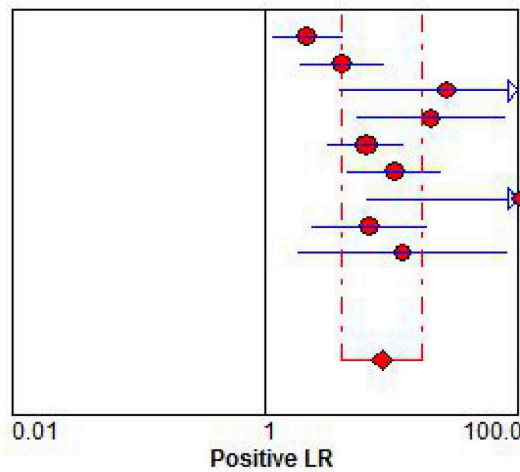

K. Dheda(2009) M. Losi (2007) Li-Ta Keng(2013)

Fei Liu(2013)

Lifan Zhang(2013)

Fei Liu(2014)

Mingfeng Liao(2013)

L.-N. Lee(2008)

Ji Young Kang(2012)
Positive LR (95\% Cl)

$2.15(1.14-4.06)$

$3.99 \quad(1.84-8.63)$

$27.14 \quad(3.81-193.12)$

$20.22 \quad(5.22-78.28)$

$6.22(3.11-12.45)$

$10.43(4.50-24.19$

$99.39 \quad(6.30-1,568.11)$

$6.63(2.31-19.01)$

$12.14(1.81-81.58)$

Random Effects Mode

Pooled Positive LR $=8.21$ ( 4.00 to 16.84 )

Cochran- $\mathrm{Q}=32.77 ; \mathrm{df}=8(\mathrm{p}=0.0001)$

Inconsistency (l-square) $=75.6 \%$

Tau-squared $=0.7983$

Figure 3. Forest plot of estimates of PLR for the pleural fluid ELISPOT assay for the diagnosis of tuberculous pleurisy. Point estimates of PLR from each study are shown as solid circles, whose size reflects the total number of cases and controls. Error bars show $95 \% \mathrm{Cls}$. Numbers indicate the reference numbers of studies.

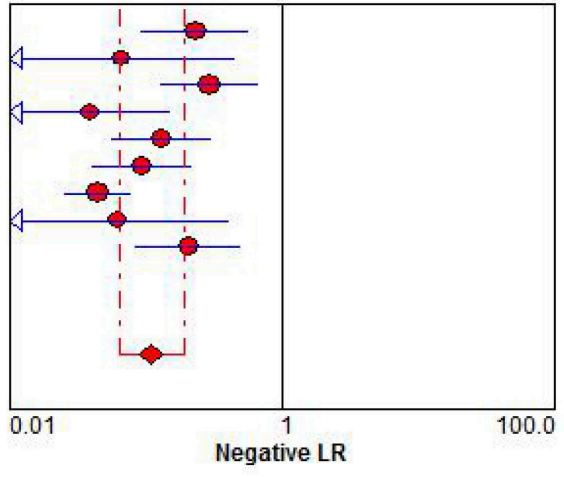

$\begin{array}{lll}\text { K. Dheda(2009) } & 0.23 & (0.09-0.58) \\ \text { M. Losi(2007) } & 0.07 & (0.01-0.45) \\ \text { Li-Ta Keng(2013) } & 0.29 & (0.13-0.67) \\ \text { Fei Liu(2013) } & 0.04 & (0.01-0.15) \\ \text { Lifan Zhang(2013) } & 0.13 & (0.06-0.30) \\ \text { Fei Liu(2014) } & 0.09 & (0.04-0.22) \\ \text { Mingfeng Liao(2013) } & 0.04 & (0.03-0.08) \\ \text { L.-N. Lee(2008) } & 0.06 & (0.01-0.42) \\ \text { Ji Young Kang(2012) } & 0.20 & (0.08-0.50)\end{array}$

Random Effects Model

Pooled Negative LR $=0.11(0.06$ to 0.19$)$

Cochran $-\mathrm{Q}=23.36 ; \mathrm{df}=8(\mathrm{p}=0.0029)$

Inconsistency (I-square) $=65.7 \%$

Tau-squared $=0.4275$

Figure 4. Forest plot of estimates of NLR for the pleural fluid ELISPOT assay for the diagnosis of tuberculous pleurisy. Point estimates of NLR from each study are shown as solid circles, whose size reflects the total number of cases and controls. Error bars show $95 \% \mathrm{Cls}$. Numbers indicate the reference numbers of studies.

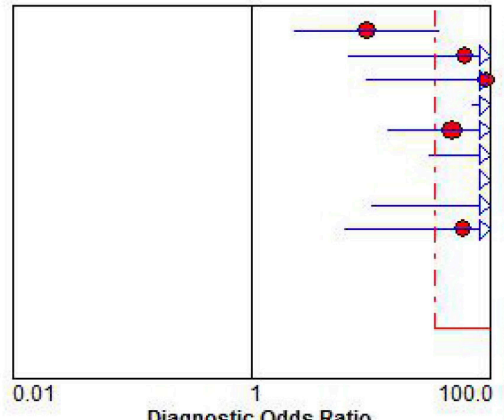

Diagnostic Odds Ratio
Diagnostic OR $(95 \% \mathrm{Cl})$

$9.30 \quad(2.29-37.70)$

$60.80 \quad(6.42-575.52)$

$92.50 \quad(9.27-922.58)$

$520.00 \quad(70.17-3,853.26)$

$48.00 \quad(14.08-163.66)$

$112.32(30.71-410.81)$

$2,220.68 \quad(129.44-38,099.24)$

$108.00(10.24-1,138.79)$

$59.50 \quad(5.95-595.04)$

Random Effects Model

Pooled Diagnostic Odds Ratio $=88.26$ (33.81 to 230.43 )

Cochran- $Q=19.11 ; \mathrm{df}=8(\mathrm{p}=0.0143)$

Inconsistency $(1-$-square $)=58.1 \%$

Tau-squared $=1.1762$

Figure 5. Forest plot of estimates of DOR for the pleural fluid ELISPOT assay for the diagnosis of tuberculous pleurisy. Point estimates of DOR from each study are shown as solid circles, whose size reflects the total number of cases and controls. Error bars show $95 \% \mathrm{Cls}$. Numbers indicate the reference numbers of studies. 


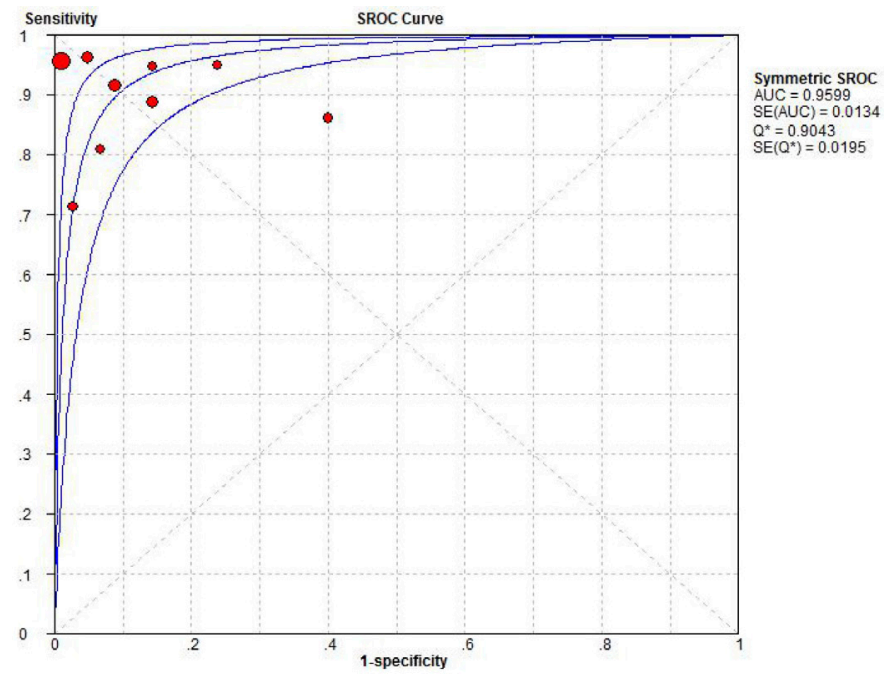

Figure 6. SROC curves for the pleural fluid ELISPOT assay. Each study is depicted as a solid circle, whose size reflects the total number of cases and controls.

SROC curves were generated by plotting sensitivity against ( 1 - specificity) for the studies included (Figure 6). All SROC curves were located near the desirable upper left corner, and the maximum joint sensitivity and specificity value was 0.96 . The high values for the area under the SROC curve and for DOR indicate a relatively high accuracy.

\section{Publication bias}

Funnel plots showed asymmetry (Figure 7), suggesting a publication bias. This was further supported by the $\mathrm{P}$ value of 0.03 for the Deeks test.

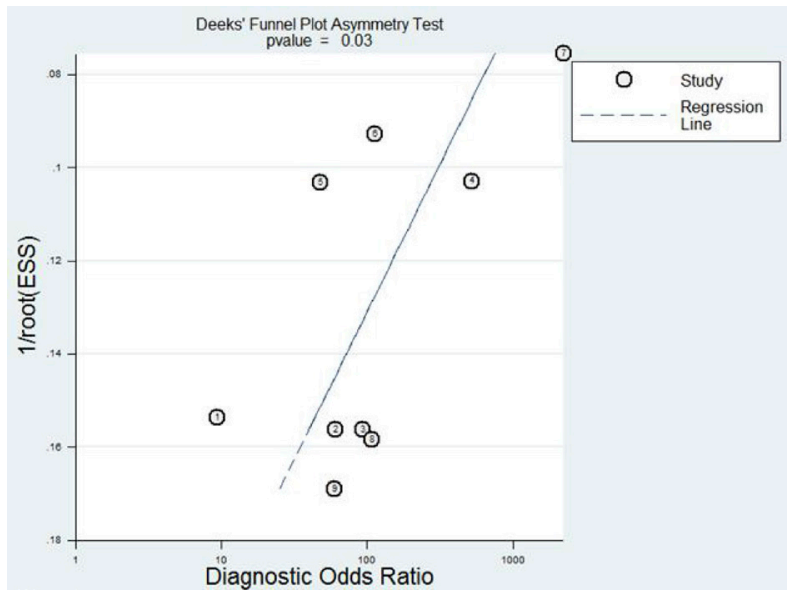

Figure 7. Funnel plot for evaluating publication bias among the nine studies included in the meta-analysis. The log DOR value was plotted against the standard error of the log DOR value; the latter serves as an indicator of sample size. Each article is shown as a solid circle, and the regression line is shown. 


\section{DISCUSSION}

The ELISPOT assay of pleural fluid may offer a method for the diagnosis of tuberculous pleurisy that is significantly faster, less invasive, and more reliable than standard approaches. Our meta-analysis of available studies on diagnostic accuracy suggests that the pleural fluid ELISPOT assay is sufficiently sensitive (0.93) and specific (0.90) to diagnose tuberculous pleurisy on its own.

Meta-analysis showed good values for all ELISPOT diagnostic accuracy parameters that we tested. DOR, which is often used as a summary indicator of test accuracy (Glas et al., 2003), combines sensitivity and specificity; it is calculated as the ratio of the proportion of positive test results in people with disease relative to the proportion of positive test results in people without disease (Liang et al., 2008). Meta-analysis of the nine studies gave a pooled DOR of 88.26, indicating a high diagnostic value. The form of the SROC curve and the area underneath it indicate the tradeoff between sensitivity and specificity; these parameters are also used to provide summarize a test performance (Liang et al., 2008). Meta-analysis showed the ELISPOT assay sensitivity to be 0.93 , with the specificity of 0.90 , maximum joint sensitivity and specificity value of 0.95 , and the area under the SROC curve of 0.9599. All these results indicate a high level of accuracy.

Since the DOR value and SROC curve analysis are difficult to translate directly into clinical terms (Deeks, 2001), diagnostic accuracy is often assessed using PLR and NLR (Deeks, 2001; Hess et al., 2008). The pooled PLR value of 8.21 suggests that patients with tuberculous pleurisy are approximately 8-fold more likely to give a positive ELISPOT test result than patients without it. This difference suggests that the ELISPOT assay can be used on its own to diagnose tuberculous pleurisy, and to allow a clinician to decide whether to initiate or continue antituberculosis treatment in patients with tuberculous pleurisy. At the same time, the pooled NLR was 0.11 , indicating that patients who give negative ELISPOT test results still have an $11 \%$ chance of having tuberculous pleurisy. This indicates that the assay is adequate on its own for ruling out tuberculous pleurisy and for deciding whether to deny or discontinue antituberculosis therapy.

Besides the ELISPOT assay, assays based on adenosine deaminase or gamma interferon have been developed to provide a similarly rapid and non-invasive diagnosis of tuberculous pleurisy. Our meta-analysis suggests that the ELISPOT assay may be more accurate than both other assays. One study (Gui and Xiao, 2014) examined the diagnostic performance of an assay based on adenosine deaminase and reported the following values: sensitivity, $0.86(95 \% \mathrm{Cl}=0.84$ to 0.88$)$; specificity, $0.88(95 \% \mathrm{Cl}=0.86$ to 0.90$)$; PLR, $6.32(95 \% \mathrm{Cl}=4.83$ to 8.26$)$; $\mathrm{NLR}, 0.15(95 \% \mathrm{Cl}=$ 0.11 to 0.22$)$; and DOR, $45.25(95 \% \mathrm{Cl}=27.63$ to 74.08$)$. A study of the diagnostic performance of an assay based on gamma interferon reported the following values: sensitivity, $0.89(95 \% \mathrm{Cl}=0.87$ to 0.91$)$; specificity, $0.97(95 \% \mathrm{Cl}=0.96$ to 0.98$)$; PLR, $23.45(95 \% \mathrm{Cl}=17.31$ to 31.78$)$; $\mathrm{NLR}, 0.11$ $(95 \% \mathrm{Cl}=0.07$ to 0.16$)$; and DOR, $272.7(95 \% \mathrm{Cl}=147.5$ to 504.2$)$ (Yamada et al., 2001). Future studies should perform side-by-side comparisons of these three assays to determine whether they can be used interchangeably or whether one should be preferred over another.

Although we conducted this meta-analysis according to widely accepted best practices, including systematic literature searching and article selection by two reviewers working independently, we did see evidence of publication bias. This may reflect the relatively small number of studies included and the fact that we included only full-length articles published in English. Even among our carefully selected articles, we found significant heterogeneity for all the diagnostic accuracy parameters that we examined, highlighting the need for larger, more rigorous studies of the ELISPOT assay. 
The available evidence suggests that the pleural fluid ELISPOT assay is accurate for the diagnosis of tuberculous pleurisy and for guiding treatment decisions. While our meta-analysis suggests that the ELISPOT assay is sufficiently accurate to be used on its own, the realities of clinical practice may argue for combining this test with other tests and with detailed analysis of patient history and clinical manifestations in order to ensure the most reliable diagnosis.

\section{Conflicts of interest}

The authors declare no conflict of interest.

\section{REFERENCES}

Aktogu S, Yorgancioglu A, Cirak K, Köse T, et al. (1996). Clinical spectrum of pulmonary and pleural tuberculosis: a report of 5,480 cases. Eur. Respir. J. 9: 2031-2035.

Chen X-Z, Zhang WH, Yang K, Zhang B, et al. (2014). Quantitative comparisons of summary receiver operating characteristics (sROC) curves among conventional serological tumor biomarkers for predicting gastric cancer in Chinese population. Tumor Biol. 35: 9015-9022.

Deeks JJ (2001). Systematic reviews in health care: systematic reviews of evaluations of diagnostic and screening tests. BMJ 323: 157-162.

Deeks JJ, Macaskill P and Irwig L (2005). The performance of tests of publication bias and other sample size effects in systematic reviews of diagnostic test accuracy was assessed. J. Clin. Epidemiol. 58: 882-893.

Dheda K, van Zyl-Smit RN, Sechi LA, Badri M, et al. (2009). Utility of quantitative T-cell responses versus unstimulated interferon- $\gamma$ for the diagnosis of pleural tuberculosis. Eur. Respir. J. 34: 1118-1126.

Glas AS, Lijmer JG, Prins MH, Bonsel GJ, et al. (2003). The diagnostic odds ratio: a single indicator of test performance. J. Clin. Epidemiol. 56: 1129-1135.

Gui X and Xiao H (2014). Diagnosis of tuberculosis pleurisy with adenosine deaminase (ADA): a systematic review and metaanalysis. Int. J. Clin. Exp. Med. 7: 3126-3135.

Hess EP, Thiruganasambandamoorthy V, Wells GA, Erwin P, et al. (2008). Diagnostic accuracy of clinical prediction rules to exclude acute coronary syndrome in the emergency department setting: a systematic review. CJEM 10: 373-382.

Kang JY, Rhee CK, Kang NH, Kim JS, et al. (2012). Clinical utility of two interferon-gamma release assays on pleural fluid for the diagnosis of tuberculous pleurisy. Tuberc. Respir. Dis. 73: 143-150.

Keng L-T, Shu CC, Chen JY, Liang SK, et al. (2013). Evaluating pleural ADA, ADA2, IFN- $\gamma$ and IGRA for diagnosing tuberculous pleurisy. J. Infect. 67: 294-302.

Lee LN, Chou CH, Wang JY, Hsu HL, et al. (2009). Enzyme-linked immunospot assay for interferon-gamma in the diagnosis of tuberculous pleurisy. Clin. Microbiol. Infect. 15: 173-179.

Liang Q-L, Shi HZ, Wang K, Qin SM, et al. (2008). Diagnostic accuracy of adenosine deaminase in tuberculous pleurisy: a meta-analysis. Respir. Med. 102: 744-754.

Liao M, Yang Q, Zhang J, Zhang M, et al. (2014). Gamma interferon immunospot assay of pleural effusion mononuclear cells for diagnosis of tuberculous pleurisy. Clin. Vaccine Immunol. 21: 347-353.

Liu F, Gao M, Zhang X, Du F, et al. (2013). Interferon-gamma release assay performance of pleural fluid and peripheral blood in pleural tuberculosis. PLoS One 8: e83857.

Liu F, Zhang X, Du F, Pan L, et al. (2014). Comparison of interferon-gamma release assays and adenosine deaminase of pleural fluid for the diagnosis of pleural tuberculosis. Zhonghua Jie He He Hu Xi Za Zhi 37: 323-327.

Losi M, Bossink A, Codecasa L, Jafari C, et al. (2007). Use of a T-cell interferon-gamma release assay for the diagnosis of tuberculous pleurisy. Eur. Respir. J. 30: 1173-1179.

Losi M, Bocchino M, Matarese A, Bellofiore B, et al. (2010). Role of the quantiferon-TB test in ruling out pleural tuberculosis: a multi-centre study. Int. J. Immunopathol. Pharmacol. 24: 159-165.

Müller R, Roberts CA and Brown TA (2014). Genotyping of ancient Mycobacterium tuberculosis strains reveals historic genetic diversity. Proc. Biol. Sci. 281: 20133236.

Pérez-Rodriguez E and Jiménez Castro D (2000). The use of adenosine deaminase and adenosine deaminase isoenzymes in the diagnosis of tuberculous pleuritis. Curr. Opin. Pulmon. Med. 6: 259-266.

Porcel JM (2009). Tuberculous pleural effusion. Lung 187: 263-270.

Trajman A, Pai M, Dheda K, van Zyl Smit R, et al. (2008). Novel tests for diagnosing tuberculous pleural effusion: what works 
and what does not? Eur. Respir. J. 31: 1098-1106.

Whiting P, Rutjes AW, Reitsma JB, Bossuyt PM, et al. (2003). The development of QUADAS: a tool for the quality assessment of studies of diagnostic accuracy included in systematic reviews. BMC Med. Res. Methodol. 3: 25.

World Health Organization (2009). Global tuberculosis control: epidemiology, strategy, financing. WHO Report 2009. World Health Organization, Geneva.

Yamada Y, Nakamura A, Hosoda M, Kato T, et al. (2001). Cytokines in pleural liquid for diagnosis of tuberculous pleurisy. Respir. Med. 95: 577-581.

Zhang L, Zhang Y, Shi X, Zhang Y, et al. (2014). Utility of T-cell interferon- $\gamma$ release assays for diagnosing tuberculous serositis: a prospective study in Beijing, China. PLoS One 9: e85030. 